\title{
Telemedicine application to headache: a critical review
}

\author{
Emanuele Spina ${ }^{1}$ (D) Gioacchino Tedeschi ${ }^{2}$ - Antonio Russo ${ }^{3} \cdot$ Francesca Trojsi $^{2} \cdot$ Rosa lodice $^{1} \cdot$ Stefano Tozza $^{1}$. \\ Aniello lovino ${ }^{1}$. Francesco lodice ${ }^{4}$. Gianmarco Abbadessa ${ }^{2} \cdot$ Francesco di Lorenzo $^{5}$. Giuseppina Miele ${ }^{3}$. \\ Elisabetta Maida ${ }^{2}$ - Giovanni Cerullo ${ }^{6}$. Maddalena Sparaco ${ }^{3} \cdot$ Marcello Silvestro $^{3}$ - Letizia Leocani ${ }^{7,8}$.

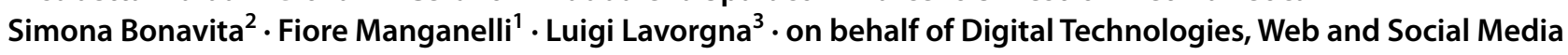 \\ Study Group of the Italian Society of Neurology (SIN)
}

Received: 30 November 2021 / Accepted: 19 January 2022 / Published online: 24 January 2022

(c) The Author(s) 2022

\begin{abstract}
Background Migraine affects more than a billion people all over the world and requires critical employment of healthcare resources. Telemedicine could be a reasonable tool to manage people suffering from headaches, and it received a big push from the COVID-19 pandemic.

Objective This review aims to propose a practical approach for the virtual management of these patients.

Methods To do this, we conducted a literature search, including 32 articles relevant to the topic treated in this review.

Results The most challenging step in telemedicine applied to practical neurology remains the clinical assessment, but through a careful headache history and a recently proposed entirely virtual neurological assessment, this hitch can be easily overcome. Electronic diary compilations and virtual administration of disability-measuring scales, conversely, are the key features of effective long-term follow-up although we do not have apps that met the criteria of scientific reliability. Furthermore, telerehabilitation seems to be effective and has demonstrated to be a solution to alternatively treat chronic patients at home, and can be considered part of the remote management of headache patients. Moreover, virtual management of headaches finds an application in specific communities of patients, as pediatric patients and for rural communities of low- and middle-income countries suffer from health disparities, with inadequate resources and knowledge gaps.

Conclusion Telemedicine could be promising for patients with no regular or convenient access to headache specialists and seems to be a priority in managing migraine patients to avoid non-urgent hospitalizations
\end{abstract}

Keywords Telemedicine $\cdot$ Tele-rehabilitation $\cdot$ Telehealth $\cdot$ Headache $\cdot$ Migraine

\section{Key points}

- With the physical distancing imposed by the COVID-19 pandemic, telemedicine could be an appealing alternative to maintain close physician-patient relation.

- Headache could be satisfactorily managed with tele-medicine. - During the first visit, the main purpose is to rule out a secondary headache with careful anamnesis, research of red flags, and gross neurological deficit.

- Synchronous and asynchronous monitoring allow physicians to control the treatments efficacy and patients' compliance, also through electronic support or mobile apps.

- Tele-rehabilitation is a promising and expanding field, both as a physical and behavioral support.

Emanuele Spina

ema.spina@libero.it

Extended author information available on the last page of the article

\section{Introduction}

Migraine affects more than a billion of people all over the world representing the second disease for years lived with disability, the first one in people under 50 years of age [1] requiring a critical employment of healthcare resources. However, since coronavirus disease 2019 (COVID-19) progressively spread around the world, and, even more since March 11, 2020, when the World Health Organization declared COVID-19 as a pandemic, human and organizational resources-once dedicated to migraine-have been redeployed to COVID-19 management. Therefore, migraine consultations have been cancelled, postponed, or converted into telemedicine. During COVID-19 pandemic, it became mandatory to find alternative strategies for patient management, as telemedicine. 
Headache healthcare will occupy an important role in the future of telemedicine because people who suffer from migraine represent approximately the $28 \%$ of any neurological outpatient clinic and because headache has so many different patterns of presentation that can lead to the possibility of being pointed out by other professional figures such as internist or gynaecologist, and then addressed to the neurologist [2].

On the other hand, telemedicine is not a novelty for physicians [3-5], although its widespread use has been precluded due to some uncertainties on the real efficacy and safety in comparison to face-to-face visits, to licensing restrictions, and technological and organizational issues $[6,7]$.

Nevertheless, in the last decade, clinical studies demonstrated that patients perceive telemedicine as cost-effective and worthwhile [8] (especially when using tablet computers as an alternative to traditional telehealth technologies) [9], with satisfaction rates and outcomes similar to traditional in-person visits [10, 11]. Furthermore, with the COVID-19 pandemic, many approaches have been proposed to manage neurological patients with telemedicine; the American Academy of Neurology developed a guidance on technology best practices and regulations [12], specific for the coronavirus emergency, and suggestion on how to perform neurologic examinations remotely. Regarding headache patients, research of the American Headache Society established the need for higher frequency of remote consultations than for other neurological patients, raising the necessity of a correct approach for their virtual management [13].

The primary goal of this paper is to propose an approach based on the available literature to manage headache patients in a virtual way, from the first visit to follow-up and therapeutical management, assuming that technical needs for a correct remote interaction have been properly satisfied.

\section{Search strategy and selection criteria}

A literature search for articles from 1998 to 2021 was conducted in the databases, including PubMed and American Academy of Neurology site using the following Medical Subject Headings (MeSH) terms and key words (also in combination): "headache," "telemedicine," "telerehabilitation," "Covid-19," "e-health," and "migraine," retrieving 139 results.

There were no language restrictions. Relevant articles were identified and located individually in PubMed and American Academy of Neurology site to examine citing and cited-by articles. The final reference list was generated based on relevance to the topics covered in this review; in particular, we decided to include only articles that clearly specified in methods the visit setting, the way to assess and monitoring patients and the possibility of tele-rehabilitation, with a more specific focus on specific communities of headache-patients. We therefore excluded case reports, paper not focusing mainly on headache and those lacking of practical proposals of patient management. Thirty-two studies, answering the aim of this review, were selected and reported.

\section{First clinical assessment}

The most challenging step in telemedicine applied to practical neurology remains the clinical assessment, as neurology is a specialty still highly trusted on semeiotics and where the physical proximity is necessary (e.g. investigation of fundus oculi, reflexes, vestibular system, elicitation and identification of trigger points). In headache patients' visits, one of the first purposes is to distinguish primary (providing for about $90 \%$ of headaches) from secondary headaches that can mimic, or may co-exist along with, a primary headache. The first step is taking a good headache history, to disentangle benign from potentially worrisome headaches. In other terms, a careful anamnesis represents the main diagnostic tool allowing the identification of primary and secondary headaches [12]. The hegemonic role of clinical history in headache medicine is of particular significance overcoming the obvious limitations related to difficulties in performing a complete neurological examination in the telemedicine scenario. To rule out significant secondary causes for headache, neurologists should always keep in mind the "headache red flags": onset $>50$ years; sudden onset; headaches increasing in frequency and severity; new-onset headache in a patient with risk factors for HIV infection or cancer; signs of systemic illness; focal neurological signs or symptoms of disease (excluding aura); recent trauma [14].

Available data showed that the virtual approach is safe needing at least 20.000 remote consultations to miss one secondary headache [15]. Moreover, clinical history and specific headache patterns can adequately address neuroimaging investigations (e.g. for patients presenting with a recentonset of severe non-remitting headache). The second step is the neurological examination, during which obvious difficulties arise, despite its general feasibility with interactive audio and video remote communication between patients and physicians. Recently, Al Hussona et al. proposed a complete virtual neurological assessment [16]. This could be sufficient for headache patients in whom the aim is to exclude gross neurological deficit and, in absence of other red flags, allows physician to proceed with requests for neuroimaging (if needed) and/or therapeutic plan.

A recent survey conducted in Norway showed that neurologists define headache and epilepsy the most suitable neurological diseases for telemedicine management because in both diseases, medical history has a crucial importance in therapeutic decision compared, for example, to movement 
disorders, in which neurological evaluation usually guide therapeutic decision [17]. The only real limitation declared by author is about performing fundoscopy in headache patients but Evans et al., in the webinar of the American Academy of Neurology for implementing recommendations for Teleneurology during Covid-19, showed some smartphone-based tools to perform remote fundoscopy examination, overcoming this limitation [18]. After collection of medical history, with assessment of the frequency and pattern of headache episodes in order to divide patients in groups of different severity $(<4 ; 4-14 ;>15$ attacks/month and newly diagnosed headache), neurological examination, presence and type of preventive therapies, measurement of functional disability through the Headache Impact Test (HIT)-6, Visual analog Scale (VAS), and Migraine Disability Assessment (MIDAS) [19], the frequency of remote consultations will be established.

\section{Remote monitoring}

In the headache management, follow-up visits and monitoring of therapy response are potentially fully replaceable by implementing mobile health (mHealth) tools. Once assessed the "benign" condition and established a therapeutic plan, it is quite easy to verify the clinical progresses of headache patients. The first step is to encourage patients to note in electronic daily diaries the number and intensities of headache attacks, with duration, site (unilateral or diffuse), associated symptoms (vomiting, nausea, photo/phonophobia), grade of disability (mild, moderate, severe), triggering factors, used drugs (NSAIDS, triptans -, dosage, effectiveness) and, for women, menses. There is growing evidence supporting the role of e-diary; indeed, the use of an electronic diary and the remote monitoring for migraine and medication overuse headache patients have been shown to be superior to classic monitoring strategies (i.e. paper diary), in reducing days with headache, the use of symptomatic drugs, and in increasing adherence to treatment [20]. More recently, headache specialists from the University of Leiden proposed a new e-diary with an automatic algorithm able to identify the type of acute attacks (e.g. migraine or tension-type), number of days with headache with an automatic classification of patients according to the type of headache and severity of the disease [21]. These digital instruments allow neurologists to capture a relatively broad range of information for migrainetracking purposes and to find, in a small set of data, relevant information for the management of migraine, such as frequency of days with headache, frequency of acute medication intake and functional impairment (the so-called $3 \mathrm{Fs}$ rule) [13]. The main issue in the monitoring of headache patients is about developing mobile apps: although recently many apps have been developed to monitor migraine, the lack of robust scientific data makes their use for medical purposes less practicable [19]. Research conducted up to 2016 identified only six article supporting the use of certain apps (Smartphone Headache Diary, myWHI diary, iHeadache, MyMigraines and MyMigrainesPro, Migraine), pointing out the lack of an optimal remote access to patients' data, a key property to allow the physician to confirm or modify therapy and, eventually, in case of therapeutic failure, to question the diagnosis [22]. A very recent review evaluated the quality of available headache management apps using behavioral change techniques (BCT), representing specific and evidence-based strategies associated with increased treatment effectiveness through self-monitoring, goal setting, and personalized feedback. Quality assessment of the identified apps took into account patients' engagement, functionality, aesthetics, and information, and 16 out of 55 ranged from good (4/5) to excellent (5/5). The strength of the BCT-based approach is based on the presence of recognized, evidence-based, strategies to improve headaches and it will surely have a key role in the future management of this class of diseases; nevertheless, none of these apps has been tested in RCTs, so it is not possible to assess with certainty their effectiveness (Table 1) [23]. On the contrary, a strength in remote monitoring of migraine is represented by the scores of the MIDAS and HIT-6 questionnaires, widely used in clinical practice. These scores can be effortlessly transferred to digital platforms, by means of scientifically validated and developed apps, to share strategical information for patients' management, i.e. discussing adjunctive pharmacological or not-pharmacological preventive measures, or delivering behavioral treatments for migraine patients [24]. Recently, wearable devices have been proposed to passively monitor vital signs with the purpose to verify the presence of some migraine attack predictors or to explore the body answer to headache pain [25].

Therefore, web-based survey was an example of asynchronous telemedicine, using to obtain information about some characteristics of migraine patients: for example, a study demonstrated that, during COVID-19 pandemic period, a higher percentage of people with migraine were depressed, even higher than of people with Multiple Sclerosis (50\% vs. $43 \%, p=0.04)$ [26].

\section{Tele-rehabilitation approaches}

A rehabilitative approach, intended as a non-pharmacological long-time support, together with pharmacological therapy, can significantly improve medical condition of headache patients [25]. Tele-rehabilitation, with both behavioral and physical therapy, seems to be effective and has demonstrated to be a solution to alternatively treat chronic patients at home, but need confirmation from large, 


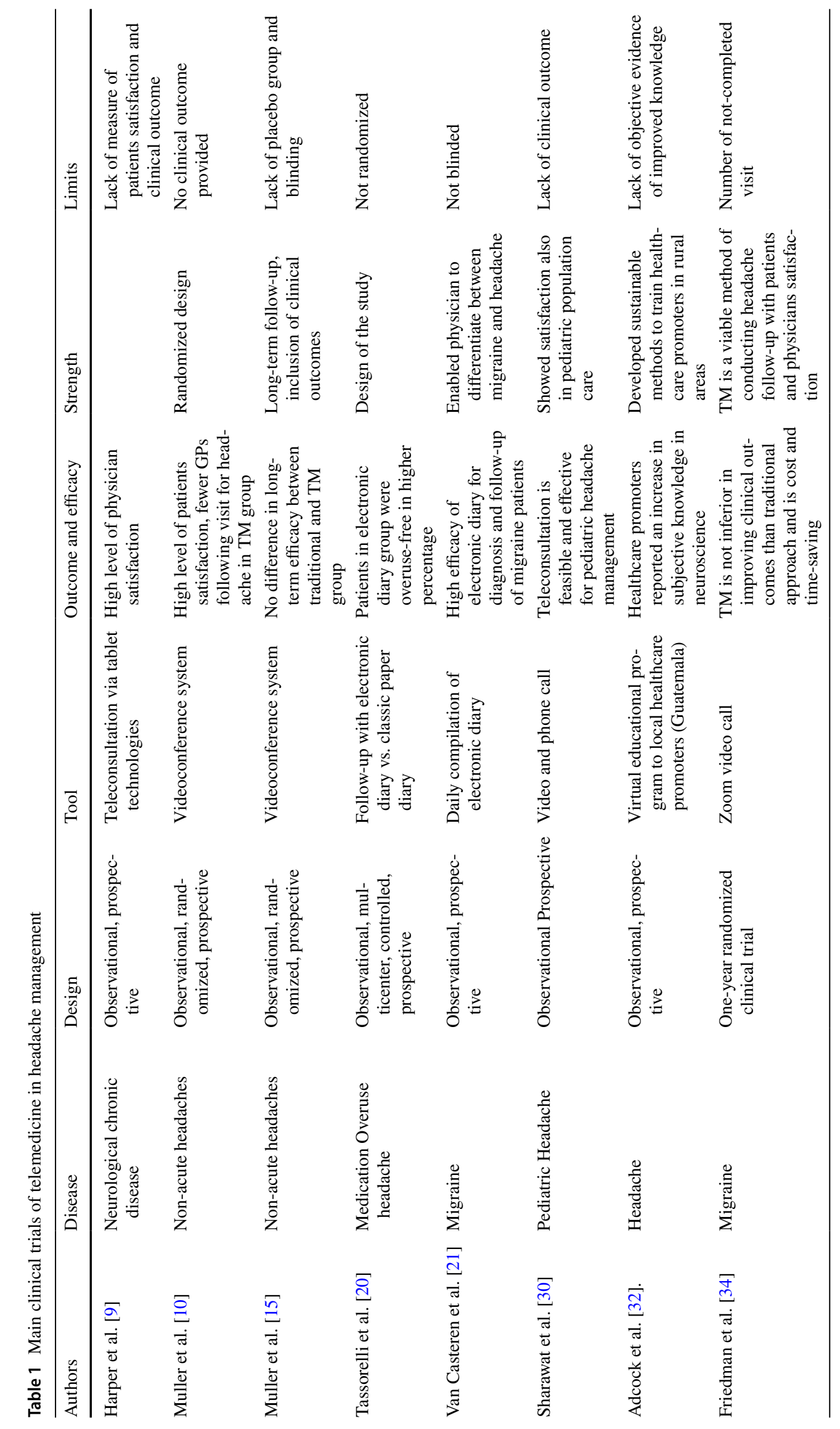


controlled clinical trials [25, 27]. As a matter of fact, there are only few applications of tele-rehabilitation to headache suffering patients [27]. Tele-rehabilitation can be considered part of the remote management of headache patients; during the follow-up visits, indeed, physician or paramedic worker should also focus on video assessment of the home environment, on patient education about pharmacotherapies (resulting in a reduction of medication-overuse headaches), lifestyle change, stress management training, cognitive therapy for pain and psychiatric comorbidities [25]. Furthermore, it has been shown that regular daily sessions of mindfulness, held by means of a smartphone application and conducted by an expert physician, have been well-accepted and effective in a cohort of chronic migraine patients with medication overuse headache [28]. Similarly, a recent narrative review pointed out the efficacy of behavioral therapy for migraine patients administered through electronic health (e-health-telecommunication-based health). On the other side, the same review showed the absence of specific mobile app (m-health) to provide these therapies [29]. This novel strategy could be incorporated into the multidisciplinary approach to migraineurs, since it may support and engage patients between outpatient visits and encourage them to play a proactive role in the management of their pain.

\section{Telemedicine experiences in specific communities}

Interestingly, telemedicine is also a convenient option for routine pediatric headache follow-up visits, resulting in high physicians, patient and family satisfaction and cost savings, reporting in 85-90\% of cases the willingness to continue the remote consulting beyond the pandemic resolution. Indeed, $90 \%$ of children were compliant with medication and $80 \%$ with lifestyle modifications without any concerns about safety [30]. Telemedicine options for children suffering from headache demonstrated to be cost-effective, saving up to 600 INR and avoiding long distance travels [31].

Regarding rural population, a recent survey conducted in Norway, apart from pointing out the high level of acceptance and feasibility (except for minor technical issues), hypothesized that telemedicine could become the standard strategy for consultations; the cost saving for each visit has been estimated in about 300 euros with savings up to 8 travelling hours [32]. Furthermore, rural communities of lowand middle-income countries suffer from health disparities, with inadequate resources and knowledge gaps. Trying to equalize these differences, a group of neurologists developed a 1-year project in a rural town of Guatemala: firstly, they identified neurological conditions that required attention through a questionnaire administered to the local population, in which people could report their main neurological complaint; afterwards, neurologists engaged local health promotors in monthly tele-lectures for 1 year, focusing on neurological troubles raised by patients, in order to improve medical assistance. The main issue complained by the local population was headache, in particular migraine, and, at the end of the year, health promotors expressed a high level of satisfaction for this program. At this moment, there are no data about the long-term impact on morbidity and mortality rates; nevertheless, telemedicine can be a way to level the existing disparities between urban and rural sectors of the world [33].

\section{Conclusion}

It has been widely demonstrated that telemedicine is equally effective compared to in-person evaluation and treatment of headache [10]. More recently, improvement in migraine disability for participants in the telemedicine group has been demonstrated not to be different from in-office patients with severe migraine-related disability [34]. Altogether, telemedicine is a feasible mode of management and an effective alternative to in-office visits for follow-up migraine care, allowing a higher physician productivity and a better patient's access because of its time and cost-effectiveness and convenience. Although controlled trials are still needed to assess the safety and effectiveness of the remote approach, a newly diagnosed patient with headache could be entirely followed up from remote with sufficient confidence.

In conclusion, telemedicine could be promising for patients with no regular or convenient access to headache specialists and seems to be a priority in managing migraine patients to avoid non-urgent hospitalizations at the time of COVID-19 pandemic, where unnecessary physical contact between physicians and patients is highly discouraged.

Acknowledgements On behalf of Digital Technologies, Web and Social Media Study Group of the Italian Society of Neurology (SIN).

\section{Declarations}

Ethical approval Authors confirm that they have read the Journal's position on issues involved in ethical publication and affirm that this report is consistent with those guidelines. This study did not directly involve human subjects.

Conflict of interest The authors declare no competing interests.

Open Access This article is licensed under a Creative Commons Attribution 4.0 International License, which permits use, sharing, adaptation, distribution and reproduction in any medium or format, as long as you give appropriate credit to the original author(s) and the source, provide a link to the Creative Commons licence, and indicate if changes were made. The images or other third party material in this article are included in the article's Creative Commons licence, unless indicated 
otherwise in a credit line to the material. If material is not included in the article's Creative Commons licence and your intended use is not permitted by statutory regulation or exceeds the permitted use, you will need to obtain permission directly from the copyright holder. To view a copy of this licence, visit http://creativecommons.org/licenses/by/4.0/.

\section{References}

1. Burch RC, Buse DC, Lipton RB (2019) Migraine: epidemiology, burden, and comorbidity. Neurol Clin. 37(4):631-649

2. Nadig R, Namapally US, Sarma KGR, Mathew T (2019) Outpatient burden of neurological disorders: a prospective evaluation of 1500 patients. Neurol India. 67(3):708-713. https://doi.org/ 10.4103/0028-3886.263249

3. Moccia M, Brigo F, Tedeschi G, Bonavita S, Lavorgna L (2018) Neurology and the internet: a review. Neu Sci 39:981-987. https://doi.org/10.1007/s10072-018-3339-9

4. Brigo F, Ponzano F, Sormani MP et al (2021) Digital work engagement among Italian neurologists. Ther Adv Chronic Dis. 12:20406223211029616. https://doi.org/10.1177/2040622321 1029616 eCollection 2021

5. Spina E, Trojsi F, Tozza S et al. How to manage with telemedicine people with neuromuscular diseases? Neurol Sci. 2021 Sep;42(9):3553-3559. Doi: 10.1007/s10072-021-05396-8. Epub 2021 Jun 25

6. Wootton R (2012) Twenty years of telemedicine in chronic disease management--an evidence synthesis. J Telemed Telecare. 18(4):211-220

7. Szperka CL, Ailani J, Barmherzig R, Klein BC, Minen MT, Halker Singh RB, Shapiro RE (2020) Migraine care in the era of COVID-19: clinical pearls and plea to insurers. Headache 60(5):833-842

8. Bonavita S, Tedeschi G, Atreja A, Lavorgna L (2020) Digital triage for people with multiple sclerosis in the age of COVID-19 pandemic. Neurol Sci. 41(5):1007-1009

9. Harper K, McLeod M, Brown SK, Wilson G, Turchan M, Gittings et al. Teleneurology service provided via tablet technology: 3-year outcomes and physician satisfaction. Rural and remote health, 19(1), (2019). 4743. Doi: 10.22605/RRH4743. Epub 2019 Mar 4

10. Müller KI, Alstadhaug KB, Bekkelund SI (2017) Telemedicine in the management of non-acute headaches: a prospective, openlabelled non-inferiority, randomized clinical trial. Cephalalgia 37(9):855-863

11. Hatcher-Martin JM, Adams JL, Anderson ER, Bove R, Burrus TM, Chehrenama et al (2020) Telemedicine in neurology: Telemedicine Work Group of the American Academy of Neurology update. Neurology 94(1):30-38

12. American academy of Neurology. Telemedicine and covid-19 implementation guide. https://www.aan.com/siteassets/homepage/tools-and-resources/practicing-neurologist\%2D\%2Dadminist rators/telemedicine-and-remote-care/20-telemedicine-and-covid 19-v103.pdf

13. Brilla R, Woo MK, Seeger SK. Do Headache patients require more care in between visits than other neurology outpatients? Headache. 2018 Jul;58(7):960-963. Doi: 10.1111/head.13339. Epub 2018 Jul 19

14. Newman LC, Lipton RB (1998 May) Emergency department evaluation of headache. Review Neurol Clin. 16(2):285-303

15. Muller KI, Alstadhaug KB, Bekkelund SI (2017) A randomized trial of telemedicine efficacy and safety for nonacute headaches.
Neurology 89(2):153-162. https://doi.org/10.1212/WNL.00000 00000004085 Epub 2017 Jun 14

16. Al Hussona M, Maher M, Chan D, Micieli JA, Kain JD, Khosravani $\mathrm{H}$, Izenberg $\mathrm{A}$, Kassardjian $\mathrm{CD}$, Mitchell $\mathrm{SB}$. The virtual neurologic exam: instructional videos and guidance for the COVID-19 era. Can J Neurol Sci. 2020 Sep;47(5):598-603. Doi: 10.1017/cjn.2020.96. Epub 2020 May 21.

17. Kristoffersen ES, Sandset EC, Winsvold BS, Faiz KW, Storstein AM. Experiences of telemedicine in neurological out-patient clinics during the COVID-19 pandemic. Ann Clin Transl Neurol. 2021 Feb; 8(2):440-447. Doi: 10.1002/acn3.51293. Epub 2020 Dec 30

18. https://www.aan.com/siteassets/home-page/tools-and-resources/ practicing-neurologist $\% 2 \mathrm{D} \% 2 \mathrm{Dadministrators} /$ telemedicine-andremote-care/20200326-telemedicine-and-covid-19-final.pdf

19. Noutsios CD, Boisvert-Plante V, Perez J, Hudon J, Ingelmo P. Telemedicine application for the evaluation of patients with nonacute headache: a narrative review. J Pain Res. 2021;14:15331542. Doi: 10.2147/JPR.S309542. eCollection 2021.

20. Tassorelli C, Jensen R, Allena M, De Icco R, Katsarava Z, Lainez JM, Leston JA, Fadic R, Spadafora S, Pagani M, Nappi $\mathrm{G}$, COMOESTAS Consortium. The added value of an electronic monitoring and alerting system in the management of medication overuse headache: a controlled multicentre study. Cephalalgia. 2017 Oct;37(12):1115-1125. Doi: 10.1177/0333102416660549. Epub 2016 Jul 20.

21. Van Casteren DS, Verhagen IE, de Boer I, de Vries Lentsch S, Fronczek R, van Zwet EW, MaassenVanDenBrink A, Terwindt GM. E-diary use in clinical headache practice: a prospective observational study. Cephalagia. 2021 Oct;41(11-12):1161-1171. Doi: https://doi.org/10.1177/03331024211010306. Epub 2021 May 2.

22. Mosadeghi-Nik M, Askari MS, Fatehi F (2016 Dec) Mobile health (mHealth) for headache disorders: a review of the evidence base. J Telemed Telecare. 22(8):472-477. https://doi.org/10.1177/13576 $33 \times 16672375$

23. Noser AE, Klages KL, Gamwell KL, Brammer CN, Hommel KA and Ramsey RR. A systematic evaluation of primary headache management apps leveraging behavior change techniques. Cephalalgia 0(0) 1-14 doi: 10.1177/03331024211053572

24. Dodick, D. W., Tepper, S. J., Lipton, R. B., Buse, D. C., Stewart, W. F., Bayliss, M. et al. Improving medical communication in migraine management: a modified Delphi study to develop a digital migraine tracker. Headache,.58(9) (2018). 1358-1372.

25. McGeary D, McGeary C. Telerehabilitation for Headache Management. Phys Med Rehabil Clin N Am. 2021 May;32(2):373391. Doi: https://doi.org/10.1016/j.pmr.2021.01.005. Epub 2021 Feb 5.

26. Matamala-Gomez M, Bottiroli S, Realdon O, Riva G, Galvagni L, Platz T, Sandrini G, De Icco R, Tassorelli C. Telemedicine and virtual reality at time of COVID-19 pandemic: an overview for future perspectives in neurorehabilitation. Front Neurol. 2021 Mar 25;12:646902. Doi: 10.3389/fneur.2021.646902. eCollection 2021.

27. Grazzi L, Rizzoli P. The adaptation of management of chronic migraine patients with medication overuse to the suspension of treatment protocols during the COVID-19 pandemic: lessons from a tertiary headache center in Milan, Italy [published online ahead of print, 2020 Apr 30]. Headache. (2020); Jul; 60(7): 1463-1464. doi: 10.1111/head.13825.

28. Stubberud A, Linde M (2018 Jul 31) Digital technology and mobile health in behavioral migrain therapy: a narrative review. Curr Pain Headache Rep. 22(10):66. https://doi.org/10.1007/ s11916-018-0718-0 
29. Rosenthal S, Yonker M (2021 Apr 12) Telemedicine in pediatric headache: a review and pratical implementation. Curr Neurol Neurosci Rep. 21(6):27. https://doi.org/10.1007/s11910-021-01112-3

30. Sharawat IK, Panda KP. Caregiver satisfaction and effectiveness of teleconsultation in children and adolescents with migraine during the ongoing COVID-19 pandemic. J Child Neurol. 2021;36(4):296-303. Doi: 10.1177/0883073820968653. Epub 2020 Nov 10.

31. Muller KI, Alstadhaug KB, Bekkelund SI (2016 May 30) Acceptability, feasibility, and cost of telemedicine for nonacute headaches: a randomized study comparing video and traditional consultations. J Med Internet Res. 18(5):e140. https://doi.org/10.2196/ jmir.5221

32. Adcock A, Frey JE. Can telemedicine address neurologic health disparities in rural Guatemala: a health promotor educational intervention study. Health Promot Int. 2021 Jun 20;daab072. Doi: 10.1093/heapro/daab072. Online ahead of print.
33. Bonavita S, Sparaco M, Russo A, Borriello G, Lavorgna L. Perceived stress and social support in a large population of people with multiple sclerosis recruited online through che COVID19 pandemic. Eur J Neurol. 2021 Oct;28(19):3396-3402. Doi: 10.1111/ene.14697. Epub 2021 Jan 18

34. Friedman DI, Rajan B, Seidmann A. A Randomized trial of telemedicine for migraine management. Cephalalgia 2019 Oct;39(12):1577-1585. Doi: 10.1177/0333102419868250. Epub 2019 Aug 26

Publisher's note Springer Nature remains neutral with regard to jurisdictional claims in published maps and institutional affiliations.

\section{Authors and Affiliations}

\section{Emanuele Spina ${ }^{1}$ (D) Gioacchino Tedeschi ${ }^{2}$ - Antonio Russo ${ }^{3} \cdot$ Francesca Trojsi $^{2} \cdot$ Rosa lodice $^{1} \cdot$ Stefano Tozza $^{1}$. Aniello lovino ${ }^{1}$. Francesco lodice ${ }^{4}$. Gianmarco Abbadessa ${ }^{2}$. Francesco di Lorenzo ${ }^{5}$. Giuseppina Miele ${ }^{3}$. Elisabetta Maida ${ }^{2} \cdot$ Giovanni Cerullo $^{6} \cdot$ Maddalena Sparaco $^{3} \cdot$ Marcello Silvestro $^{3} \cdot$ Letizia Leocani $^{7,8}$.

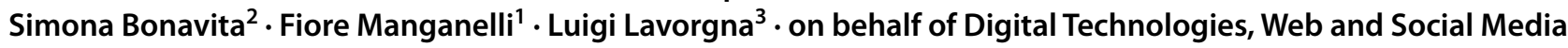 Study Group of the Italian Society of Neurology (SIN)}

1 Department of Neuroscience, Reproductive Science and Odontostomatology, University of Naples "Federico II" Via Pansini, 5, 81028 Naples, Italy

2 Department of Advanced Medical and Surgical Sciences, AOU University of Campania "Luigi Vanvitelli", Caserta, Italy

3 1st Clinic Of Neurology, AOU University of Campania "Luigi Vanvitelli,", Caserta, Italy

4 IRCSS San Raffaele Pisana, Neurology Unit, Rome, Italy
5 IRCSS Santa Lucia, Neurology Unit, Rome, Italy

6 Ospedale del Mare, Napoli, Italy

7 Experimental Neurophysiology Unit, Institute of Experimental Neurology (INSPE), San Raffaele Scientific Institute, Milan, Italy

8 Vita-Salute San Raffaele University, Milan, Italy 